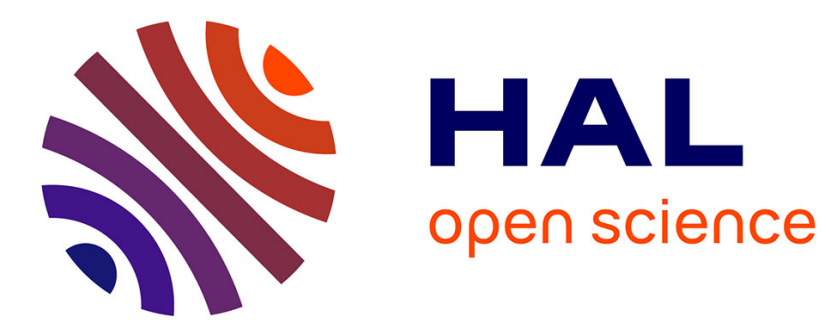

\title{
Optimal adaptive IMRT strategy to spare the parotid glands in oropharyngeal cancer
}

Pengcheng Zhang, Antoine Simon, Bastien Rigaud, Joël Castelli, Juan David

Ospina Arango, Mohamed Nassef, Olivier Henry, Jian Zhu, Pascal Haigron, Baosheng Li, et al.

\section{To cite this version:}

Pengcheng Zhang, Antoine Simon, Bastien Rigaud, Joël Castelli, Juan David Ospina Arango, et al.. Optimal adaptive IMRT strategy to spare the parotid glands in oropharyngeal cancer. Radiotherapy \& Oncology, 2016, 120 (1), pp.41-47. 10.1016/j.radonc.2016.05.028 . hal-01340279

\section{HAL Id: hal-01340279 \\ https://hal.science/hal-01340279}

Submitted on 30 Jun 2016

HAL is a multi-disciplinary open access archive for the deposit and dissemination of scientific research documents, whether they are published or not. The documents may come from teaching and research institutions in France or abroad, or from public or private research centers.
L'archive ouverte pluridisciplinaire $\mathbf{H A L}$, est destinée au dépôt et à la diffusion de documents scientifiques de niveau recherche, publiés ou non, émanant des établissements d'enseignement et de recherche français ou étrangers, des laboratoires publics ou privés. 


\section{Optimal adaptive IMRT strategy to spare the parotid glands in oropharyngeal cancer}

Pengcheng Zhang $P h D^{(1,2,3,4)}$, Antoine Simon $P h D^{(2,3,4)}$, Bastien Rigaud $M S c^{(2,3)^{*}}$, Joël Castelli $M D, M S c$ ${ }^{(2,3,5)}$, Juan David Ospina Arango $P h D^{(2,3)}$, Mohamed Nassef $M S c^{(2,3)}$, Olivier Henry $M S c^{(5)}$, Jian Zhu $P h D^{(6,7)}$, Pascal Haigron $P h D^{(2,3,4)}$, Baosheng Li $M D, P h D^{(6,7)}$, Huazhong Shu $P h D^{(4,7)}$, and Renaud De Crevoisier $M D, P h D^{(2,3,4,5)^{*}}$

1. National Key Laboratory for Electronic Measurement Technology, North University of China, Taiyuan, 030051, People's Republic of China

2. Université de Rennes 1, LTSI, Campus de Beaulieu, Rennes, F-35042, France

3. INSERM, U1099, Campus de Beaulieu, Rennes, F-35042, France

4. Centre de Recherche en Information médicale sino-français (CRIBs), Rennes, F-35042, France

5. Centre Eugene Marquis, Radiotherapy Department, Rennes, F-35000, France

6. Department of Radiation Oncology, Shandong Cancer Hospital, Jinan, People's Republic of China

7. Laboratory of Image Science and Technology, Southeast University, Nanjing, People's Republic of China

Running title: Adaptive radiotherapy strategy in oropharyngeal cancer 
* Corresponding author: email address: r.de-crevoisier@rennes.unicancer.fr or bastien.rig@gmail.com, tel.: $+33(0) 299253031$.

Conflict of Interest: the authors declare that they have no competing interests.

Acknowledgments: part of this work has received a grant from the French INCa within the PAIR VADS program. This work was partly supported by the French ANR within the Investissement d'Avenir program (Labex CAMI) under reference ANR-11-LABX-0004 and by the French "Ligue contre le Cancer". 
Keywords: head and neck, oropharyngeal, radiotherapy, replanning 


\begin{abstract}
Purpose

In oropharyngeal cancer adaptive radiation therapy (ART), this study aimed to quantify the dosimetric benefit of numerous replanning strategies, defined by various numbers and timings of replannings, with regard to parotid gland (PG) sparing.
\end{abstract}

\title{
Material and methods
}

Thirteen oropharyngeal cancer patients had one planning and then six weekly CT scans during the seven weeks of IMRT. Weekly doses were recalculated without replanning or with replanning to spare the PG. Sixty-three ART scenarios were simulated by considering all the combinations of numbers and timings of replanning. The PG cumulated doses corresponding to "standard" IMRT and ART scenarios were estimated and compared, either by calculating the average of weekly doses or using deformable image registration (DIR).

\section{Results}

Considering average weekly doses, the mean PG overdose using standard IMRT, compared to the planned dose, was 4.1 Gy. The mean dosimetric benefit of 6 replannings was 3.3 Gy. Replanning at weeks 1, 1-5, 1-2-5, 1-2-4-5 and 1-2-4-5-6 produced the lowest PG mean doses, 94\% of the maximum benefit being obtained with 3 replannings. The percentage of patients who had a benefit superior to 5 Gy for the contralateral PG was $31 \%$ for the three-replannings strategy. The same conclusions were found using DIR.

\section{Conclusion}

Early replannings proved the most beneficial for PG sparing, three replannings (weeks 1-2-5), representing an attractive combination for ART in oropharyngeal cancer. 


\section{Introduction}

Intensity-modulated radiation therapy (IMRT) has demonstrated its efficiency in head and neck cancer (HNC) by delivering highly-conformal doses to the tumor whilst sparing the organs at risk (OARs) [1-3], particularly the parotid glands (PGs). Large anatomical variations can, however, occur during the treatment, such as weight loss [4,5], primary tumor shrinking [4], parotid gland (PG) volume reduction [6], and neck diameter reduction [7-9]. These modifications may generate differences between the planned and actual delivered doses, inducing the risk of PG radiation overdose $[10,11]$ that can potentially increase the risk of xerostomia [12].

Adaptive radiotherapy (ART), based on replanning(s) during the treatment, has proven able to decrease the PG mean dose during HNC IMRT [5], yet the optimal number and timing of these replannings are, to date, unclear. Different ART scenarios have been investigated and compared to a classical non-ART scenario with varying numbers and timings tested: one replanning performed either mid treatment [13], at week 2 [14] or at a patient-specific time based on weight loss or tumor shrinkage [10], two replannings at weeks 2 and $4[13,15]$, three replannings at the $9^{\text {th }}, 19^{\text {th }}$, and $29^{\text {th }}$ fractions [16], and, the most extensive ART strategy, six weekly replannings $[8,13]$. All these studies show that ART results in a decrease of the PG mean dose. However, since the procedure of replanning is time consuming [17-19], the most optimal replanning strategy, i.e. the optimal number and timing of replanning, still requires thorough assessment.

This study, focused on IMRT for oropharyngeal cancer, aimed to determine the best ART scenario, namely to assess the optimal number and timing of replanning for PG sparing.

\section{Materials and methods}




\subsection{Patients and tumors}

The study enrolled 13 patients with a mean age of 64 years (range: 50-76 years). All tumors were locally advanced oropharyngeal carcinomas (Stage III or IV, AJCC 7th ed). The PG mean volume was 25.9cc (range: 16.6-52.2 cc). Three ipsilateral PGs were completely included within the planning target volume (PTV), thus excluded from the study, resulting in a total of 23 PGs analyzed (13 contralateral and 10 ipsilateral PGs). Table 1 shows the patient and tumor characteristics.

\subsection{Treatment and planning}

All patients underwent IMRT, receiving a total dose of 70 Gy (2 Gy/fraction, 35 fractions), with a simultaneous integrated boost technique [17] and concomitant chemotherapy. Planning computed tomography (CT0) was acquired with intravenous contrast agents using $2 \mathrm{~mm}$ slice thickness, from the vertex to the carina. A thermoplastic head and shoulder mask with five fixation points was used. Positron emission tomography (PET) and magnetic resonance imaging (MRI) co-registration was used for tumor delineation. The gross tumor volume (GTV) corresponded to the primary tumor along with involved lymph nodes. Three target volumes were generated for each patient. A clinical target volume receiving 70 Gy $\left(\mathrm{CTV}_{70}\right)$ was equal to the GTV plus a 5mm 3D margin, which was adjusted to exclude any air cavities and bone mass showing no evidence of tumor invasion. $\mathrm{CTV}_{63}$ corresponded to the area at high-risk of microscopic spread, while $\mathrm{CTV}_{56}$ corresponded to the low-risk subclinical area. GTV, $\mathrm{CTV}_{63}, \mathrm{CTV}_{56}$, and all organs at risk were manually delineated on each CT slice by a single radiation oncologist. The PTVs were generated by adding a 5mm 3D margin around the CTVs. PTV expansion was limited to $3 \mathrm{~mm}$ from the skin surface in order to avoid part of the build-up region and limit skin toxicity [20]. All IMRT plans were generated using Pinnacle V9.2. Seven coplanar 6-MV photon beams were employed with a step-andshoot IMRT technique. The prescribed doses were 70 Gy to the PTV 70,63 Gy to PTV 63 , and 56 Gy to PTV $_{56}$. The collapsed cone convolution/superposition algorithm was used for dose calculation. The 
maximum dose (D2\%) within the PTV was $110 \%$ of the prescribed dose. The minimum PTV volume covered by the $95 \%$ isodose was $95 \%$. Dose constraints were set according to the RTOG recommendations [21] as follows: spinal cord: maximum dose $<45 \mathrm{~Gy}$, brainstem: maximum dose $<54$ Gy, optic nerves: maximum dose $<54 \mathrm{~Gy}$, contralateral parotid: mean dose $<30 \mathrm{~Gy}$, median dose $<26$ Gy, ipsilateral parotid: mean dose as low as possible, oral cavity: mean dose $<30 \mathrm{~Gy}, \mathrm{~V} 30<65 \%$ and V35 $<$ $35 \%$ and lips: maximum dose $<30 \mathrm{~Gy}$ and mean dose $<20 \mathrm{~Gy}$. PG sparing was not allowed if it was in detriment of PTV coverage and/or of other important OARs sparing. The treatment plans were performed by a single physicist. Initial planning characteristics are shown in Table 1.

The time between CT0 acquisition and the start of the treatment was on average (range) 16 days (5-39 days). The patients were treated as planned on the CT0 and no changes were applied to the parameters of the initial planning during the treatment (the following ART scenarios were generated for the dosimetric study only). During the treatment course, weekly in-room stereoscopic imaging was used to correct any set-up errors $>5 \mathrm{~mm}$. All patients signed informed consent forms. The study was approved by the institutional review board (ARTIX study NCT01874587).

\subsection{Weekly dose estimations without replanning (non-ART scenario)}

During treatment, each patient underwent six weekly CTs (CT1 to CT6) (Figure 1) according to the same parameters as CT0, except for the intravenous contrast agents (not systematically used, particularly in case of cisplatin based chemotherapy). For each patient, the anatomical structures were manually delineated on each weekly $\mathrm{CT}$ by the same radiation oncologist without any propagation tool. For $\mathrm{CTV}_{70}$, the initial delineation at CT0 was maintained on each weekly CT with an adjustment to exclude any air cavities and bone mass showing no evidence of initial tumor invasion. When complete response was achieved, the initial macroscopically-involved areas were still included in the $\mathrm{CTV}_{70}$. The weekly doses (Figure 1, Step 
1A) were then estimated by calculating the dose distribution on the weekly CTs (CT1 to CT6), using the same treatment parameters and isocenter as those in $\mathrm{CT} 0$ and a bone-based rigid registration to simulate patient setup.

\subsection{Replanning and ART scenarios}

A new IMRT plan was generated on each weekly CT, from week 1 (CT1) to week 6 (CT6) by the same physicist than for the planning (Figure 1, Step 1C). The replanning procedure corresponded to a new planning based on the re-contoured structures, using the same objectives and dose constraints than the initial planning on $\mathrm{CT}$, and above all respecting the RTOG recommendations [21]. Parotid sparing was not allowed if it was in detriment of PTV coverage and/or of other important OARs.

All possible ART scenarios were simulated by considering 1 to 6 replannings and all possible timings. For example, for two replannings, 15 scenarios were simulated, taking into consideration the fact that replannings could occur at weeks 1 and 2 or weeks 1 and 3, and so on up to weeks 5 and 6 . Thus, by considering one to six replannings with all possible timings, a total number of 63 ART scenarios were simulated.

For each patient, each ART scenario corresponded to a series of six dose distributions calculated on the six weekly CTs. For each weekly CT, the most recent plan in the considered scenario was selected, i.e. either the initial plan or last replanning, for dose calculation. For example, in the case of two replannings at weeks 2 and 4, the following combinations of CT and plans were considered: CT1 - initial plan; CT2 replanning; CT3 - week 2 replanning; CT4 - replanning; CT5 - week 4 replanning; CT6 - week 4 replanning. 


\subsection{Parotid gland delivered dose estimation}

The cumulated dose according to the different scenarios was estimated in each PG using two different methods, calculating the average PG mean dose and by deformable image registration (DIR).

- The average PG mean dose was analyzed by calculating the sum of the PG mean doses computed on the weekly CTs and normalized according to the number of CTs.

- The cumulated dose was also computed as the sum of the dose distributions at different fractions (weekly CTs) mapped on the planning CT by considering the anatomical transformation between the fraction anatomies and planning anatomy. The anatomical transformation was estimated by delineation-based DIR. For each PG, a signed distance map was first generated to represent the internal and external squared Euclidean distance between each voxel and the PG surface. Distance maps of each PG were then registered using the Demons registration algorithm [22,23]. The resulting deformation fields were used to map the weekly dose distributions from the weekly CT to the planning CT using tri-linear interpolation. These mapped weekly dose distributions could be then added in order to calculate the cumulated dose. The Sorensen-Dice (DSC), a similarity coefficient computed between two regions [24], was used to evaluate the performance of the PG DIR. The average DSC, computed between the delineation on CT0 and the delineations on weekly CTs propagated thanks to registration, was 0.66 (range: $0.36-0.88$ ) and 0.92 (range: 0.83-0.95) after bone-based rigid registration and DIR respectively.

In total, average PG mean doses and DIR-based cumulated doses were therefore estimated for both the non-ART scenario and all ART scenarios. Each ART scenario was then compared to the non-ART scenario in terms of PG mean cumulated dose. For each ART scenario, the percentages of patients who had a benefit, compared to the non-ART scenario, superior to 4 Gy for both PGs, for at least one PG, for only the ipsilateral (IL) PG and for only the contralateral (CL) PG were computed. 


\subsection{CTV delivered dose estimation}

The average $\mathrm{CTV}_{70} \mathrm{D} 98 \%$ (minimal dose received by $95 \%$ of the $\mathrm{CTV}_{70}$ ) was analyzed by calculating the sum of the $\mathrm{CTV}_{70} \mathrm{D} 98 \%$ computed on the weekly CTs and normalized according to the number of CTs, without and with ART. The $\mathrm{CTV}_{70} \mathrm{D} 98 \%$ (minimal dose received by $98 \%$ of the $\mathrm{CTV}_{70}$ ) was also analyzed.

\subsection{Statistical analysis}

The Wilcoxon signed-rank test was first used to compare the OARs (other than the PGs) and the PTVs doses (using the RTOG indexes) of the initial planning with the fraction doses of the weekly replannings. The Wilcoxon test was also carried out to compare the PG and $\mathrm{CTV}_{70} \mathrm{D} 98 \%$ doses of each ART scenario with the doses of the non-ART scenario. Statistical analysis was carried out using the R language and environment for statistical computing.

\section{Results}

$\mathrm{CTV}_{70}$ and PG volume differences over the course of the treatment (between CT0 and CT6) are shown Table 1. PTVs and OARs dosimetric indexes (other than those of the PGs) did not significantly differ between the initial planning and the weekly replannings.

The optimal number and timing of replannings to spare the PG are presented first by calculating the average PG mean dose and secondly using the DIR method to cumulate the PG dose. 


\subsection{Optimal number and timing of replannings considering the average PG mean doses}

Relatively to the planning, the total number of overdosed PGs was 16 (9 CL and 7 IL), with a mean (range) overdose of 4.1 Gy (0.5 - $11.5 \mathrm{~Gy})$. Figure 2 (superior part) shows the differences between the average PG mean dose for the non-ART (or each ART scenarios) and the PG planned dose (considered as the reference). The mean (range) dose difference between the non-ART scenario and the planned dose was 1.7 Gy (-6.8 - 11.5 Gy) (red line in Figure 2).

Relatively to the non-ART scenario, the mean and maximum dosimetric benefits of one to six replannings (highest benefit for a given number of replannings) considering both PGs were 2.2 Gy and 10 Gy with one replanning (week 1), 2.9 Gy and 10.6 Gy with two replannings (weeks 1 and 5), 3.1 Gy and 11.0 Gy with three replannings (weeks 1, 2 and 5), 3.2 Gy and 10.8 Gy with four replannings (weeks 1, 2, 4 and 5), 3.3 Gy and 10.8 Gy with five replannings (weeks 1,2, 4, 5 and 6) and 3.3 Gy and 10.8 Gy with six replannings (weeks 1 to 6), respectively. The dosimetric benefit of each of these ART scenarios for the CL and IL PGs is detailed in Table 2. Considering the maximum benefit obtained by six replannings, $94 \%$ of this benefit was therefore obtained with only three replannings, conducted at weeks 1, 2 and 5 .

Figure 2 (inferior part) presents the percentages of patients with a benefit higher than 4 Gy for all the scenarios and for at least one PG (IL or CL), both PGs (IL and CL), the CL PG and the IL PG. Figure 3 presents the percentage of patients with a PG benefit of more than 0 to 12 Gy for the three-replannings scenario (weeks 1-2-5).

3.2 Optimal number and timing of replannings considering the DIR-based cumulated PG doses 
Relatively to the planning, the total number of overdosed PGs was 15 (9 CL and 6 IL), with a mean overdose of 3.8 Gy $(0.6-9.6$ Gy). Figure 4 (superior part) (supplementary material) shows the differences between the cumulated dose (for the non-ART and each ART scenarios) and the planned dose for all the PGs. The mean (range) dose difference between the non-ART scenario and the planned dose was 1.2 Gy (8.1 - 9.6 Gy) (red line in Figure 4).

Relatively to the non-ART scenario, the mean and maximum dosimetric benefits of one to six replannings (highest benefit for a given number of replannings) considering both PGs were 2.2 Gy and 10.4 Gy with one replanning (week 1), 3.0 Gy and 10.9 Gy with two replannings (weeks 1 and 5), 3.1 Gy and 11.8 Gy with three replannings (weeks 1,2 and 5), 3.3 Gy and 11.3 Gy with four replannings (weeks 1, 2, 4 and 5), 3.4 Gy and 11.3 Gy with five replannings (weeks 1, 2, 4, 5 and 6) and 3.3 Gy and 11.3 Gy with six replannings (weeks 1 to 6 ), respectively.

The dosimetric benefit of each of these ART scenarios for the CL and IL PGs is detailed in Table 3 (supplementary material). Relatively to the maximum benefit obtained by six replannings, $95 \%$ of this benefit was obtained with only three replannings, performed at weeks 1,2 and 5.

Figure 4 (inferior part) (supplementary material) presents the percentages of patients with a benefit higher than 4 Gy for all the scenarios and for at least one PG (IL or CL), both PGs (IL and CL), the CL PG and the IL PG. Figure 5 (supplementary material) presents the percentage of patients with a benefit from 0 to 12 Gy for the three-replannings scenario (weeks 1-2-5).

\subsection{CTV doses without ART and with ART}

Comparing the non-ART fractions to the planning, the $\mathrm{CTV}_{70}$ was underdosed in $73 \%$ of the fractions. The mean and the maximum differences of the $\mathrm{CTV}_{70} \mathrm{D} 98 \%$ between the non-ART fractions and the planning were 1.6 Gy and 3.1 Gy, respectively. 
Comparing the ART to the non-ART fractions, increasing the number of replannings improved the $\mathrm{CTV}_{70}$ coverage, as assessed by the mean $\mathrm{CTV}_{70} \mathrm{D} 98 \%$ presented in Table 2.

\section{Discussion}

The goal of our study was to compare different scenarios of replannings with regards to PG sparing in oropharyngeal cancer while, for each fraction, keeping the same PTV coverage than at the planning. By testing all different combinations of one to six weekly replannings during the 7 weeks of treatment, producing a total of 63 scenarios, we found that the maximum PG sparing was obtained with six replannings, resulting in an average mean dose decrease of $1.6 \mathrm{~Gy}$ (6.4 Gy max.) compared to the planned dose, and of 3.3 Gy (10.8 Gy max.) compared to the average dose without ART. Considering this six replannings scenario, $54 \%$ and $23 \%$ of patients had a benefit superior to $4 \mathrm{~Gy}$ in at least one PG and in two PGs, respectively. Three replannings, performed at weeks 1-2-5, were found to decrease the PG mean dose by 3.1 Gy (11.0 Gy max.) compared to the non-ART scenario, corresponding to $54 \%$ and $15 \%$ of patients having a benefit superior to 4 Gy in at least one PG and in both PGs respectively. This scenario thus resulted to more than $90 \%$ of the benefit obtained with the maximalist six replannings approach. The ART dosimetric benefit was higher for the contralateral PGs than for the ipsilateral PGs. ART strategies were therefore compared by considering both the PG dose difference and the percentage of patients who had a clinically meaningful PG benefit (superior to 4 Gy). Due to uncertainties in the estimation of the cumulated dose received by the PGs during the whole treatment, two different methods were used, one calculating the average of the weekly mean doses and the other one based on DIR. Both methods led to the same conclusions for the identification of the best replanning strategies. It must also be pointed out that the CTV was slightly underdosed in the vast majority of fractions when using a standard IMRT 
without ART. Indeed, increasing the number of replannings also increased the coverage of the target. This correction of the tumor underdosage by ART could have however limited the PG sparring.

While different numbers of replannings aimed at sparing the PG have been investigated in the literature, the optimal timing of these replannings has not been thoroughly assessed [8,13,15,16,25-34]. The endpoint of these studies was the quantification of the dosimetric benefit of sparing the PG, while keeping or even increasing the minimum dose in the PTV, using different CT-based IMRT replanning scenarios. Only one tested the clinical impact of replanning [33]. The number of patients analyzed per study also widely varied from 10 to 86 . The number of replannings varied from one to six. The mid-treatment period, typically the third or fourth week, was generally chosen to test one [13,26,29-32] or two replannings $[15,25,27,28]$. Weekly replannings were tested in three studies $[8,13,34]$. Dose comparison was performed either at the fraction $[28,30-32,34]$ or through the estimation of the cumulated dose by deformable image registration $[8,13,25,26]$, or even by summing the dose-volume histograms [29].

Compared to the planned dose, the contra-/ipsi- lateral PG doses were found to be decreased by 0.6/1.2 Gy, and 0.8/4.1 Gy when one and two replannings were conducted, respectively [26]. Two replannings have also shown to decrease the PG median dose by $2.2-7.1 \%$ [15,27]. One mid-course replanning and six replannings resulted in decreasing the PG mean dose by 3 and 6\%, respectively [13]. A weekly replanning schedule decreased the PG mean dose by 3.6 Gy [8] or by 3\% per week [13]. Our results appear relatively similar to those in the literature for a given tested scenario. Nevertheless, one study found no benefit through performing four replannings [25]. In their results, the PG mean dose at planning, cumulated dose without replanning, and cumulated dose with replanning were $17.9 \mathrm{~Gy}, 18.7 \mathrm{~Gy}$, and $18.7 \mathrm{~Gy}$, respectively. The PG mean planned dose was thus lower in their study than in our work, probably due to the irradiated tumor site comprising only one oropharyngeal tumor. Such results suggest that the oropharyngeal site could benefit the most from an ART strategy. In total, the majority of the studies suggest that the more replannings are used, the more the PG can be spared, and especially so for oropharyngeal tumors. 
However, despite large anatomical variations, Ho et al. did not find any dose difference for the OARs in a nasopharyngeal tumor cohort [35]. This series differs however from our work again by the primary tumor (nasopharyngeal versus oropharyngeal tumor), by the smallest planned dose (65 Gy versus 70 Gy) which may impact the position of the dose gradient relatively to the PGs, and by the use of neoadjuvant TPF chemotherapy in $30 \%$ of the patients which reduced the CTV before the radiotherapy. It must also be pointed out that, in our series, the proportion of "spontaneously" underdosed PGs (range 6.8 - 1.5 Gy) without any replanning (i.e. standard IMRT) was surprisingly 30\%, which may be explained by the shrinkage of the PGs that could move them away from the high dose area.

The clinical impact of replanning was evaluated in only one series involving 86 patients with nasopharyngeal cancer [33]. One or two replannings were performed at the third and fifth weeks, with no anatomical or dosimetric selection criteria. A comparison was performed with 43 patients who refused replanning. Replanning improved both the patients' quality of life and localized disease control, yet had no impact on overall survival.

Limiting the number of replannings is a crucial issue, given the burden each supplementary replanning entails. In previous studies, the mid-treatment period or $5^{\text {th }}$ week (at the boost) was selected for one replanning [36,37]. In contrast, our results show that the beginning of the treatment (first two weeks) is the most crucial time for replanning. The CTV shrinkage is, in fact, particularly significant during this early period of radiotherapy [38], and PG overdose at the end of the treatment has been shown to be highly correlated with overdose on the first treatment day [39]. Moreover, the earlier the replanning is performed, the more it will impact on the treatment. The strong impact of the first week replanning suggests also reducing the delay between the initial planning and the beginning of treatment. While our study demonstrated that each supplementary replanning decreases the PG mean dose, three replannings at weeks 1-2-5 do, however, appear to offer a good compromise, achieving $94 \%$ of the maximum weekly replanning benefit. From a previous maximalist replanning strategy study, we found that the benefit of 5 
Gy could reduce the xerostomia risk of nearly $10 \%$ [8]. Our study motivates the use of a three-replannings strategy to reduce the doses at the CL PG of 5 Gy for $31 \%$ of our cohort, leading to a non-negligible reduction of the xerostomia risk. However, the mechanisms leading to xerostomia and its resolution are complex and remain to elucidate. A recent study has shown that an early PG shrinkage is predictive of xerostomia resolution [40], still showing the importance of the first weeks of treatment. Considering the parotid glands as a unified organ also have some limitations since some specific sub-regions of parotids, where salivary stem cells are concentrated, may be preferentially related to xerostomia [41].

This study also shows that considering the individual benefit of the replannings separately is not sufficient to assess their combinations. Indeed, the synergy between replannings at different times should also be exploited to obtain the best PG sparing. The efficiency of a given replanning is actually related to the combination of two factors: (i) the anatomical modifications it will enable to compensate with respect to the previous (re-)plan and (ii) the duration this new plan will be used, i.e. corresponding to the number of fractions the new plan will be applied until the end of the treatment or a new replanning.

Another way to limit the number of replannings could be a more patient-specific approach based on anatomical or dosimetric predictors of PG overdose [42]. Ideally, these criteria should be defined on both pre-treatment planning CT and per-treatment cone-beam CT (CBCT). Due to the poor image quality, limited field of view and complexity of the dose calculation, such CBCT based approach has however not been yet solved. We therefore opted for weekly CT scans for dose calculation, also assuming that the anatomy at the fraction was representative of the anatomy of the whole week of treatment. We also simulated the patient setup by a bone-based rigid registration, neglecting therefore the setup error.

The main limitation of this study is the small number of patients. The results may also depend however of the full IMRT procedure (delineation, IMRT technique, dose constrains and objectives, timing of the CTs...). The conclusion of our study should be therefore considered carefully, with a need for an external validation cohort to confirm the results. Our monocentric study appears nevertheless homogeneous since 
comprising oropharyngeal tumors only, a single radiation oncologist for all the delineations and a single physicist for the (re)plannings. Moreover, the PG cumulated doses have been estimated by using both a mean dose approach and an elastic registration method, leading to concordant statistically significant conclusions. Finally, if the cumulated doses to the target and the OARs (other than the PGs) were not estimated, the PTV coverage and OARs sparing were not significantly degraded between the initial plannings and the replannings. Clinical finding should finally confirm the pertinence of the ART strategy.

\section{Conclusion}

Three replannings conducted at weeks 1,2 and 5 appear to offer a pragmatic and attractive ART strategy enabling a decrease of the PG dose by 3.1 Gy in locally-advanced oropharyngeal carcinoma, while also increasing slightly the target coverage. Clinical trials are, however, needed to demonstrate the benefit of this approach in terms of decreasing the risk of xerostomia while keeping the same local control. 
Table 1: Patient, tumor and initial planning (CT0) characteristics and volume differences over the course of the treatment (between CT0 and CT6)

\begin{tabular}{|c|c|c|c|c|c|c|c|c|c|c|c|}
\hline \multirow[t]{2}{*}{ ID } & \multirow{2}{*}{ Gender } & \multirow{2}{*}{ Age } & \multirow{2}{*}{ TNM } & \multicolumn{3}{|c|}{ Volume at CTO (cc) } & \multicolumn{2}{|c|}{$\begin{array}{c}\text { Dmean at CT0 } \\
\text { (Gy) }\end{array}$} & \multicolumn{3}{|c|}{$\begin{array}{c}\text { Volume variation between CT0 } \\
\text { and CT6 (in \%) }\end{array}$} \\
\hline & & & & $\mathrm{CTV}_{70}$ & ILP & CLP & ILP & CLP & $\mathrm{CTV}_{70}$ & ILP & CLP \\
\hline 1 & $\mathrm{M}$ & 76 & T3N1 & 45.2 & 52.1 & 48.6 & 30.2 & 31.1 & -53.1 & -10.0 & -55.0 \\
\hline 2 & $\mathrm{~F}$ & 63 & $\mathrm{~T} 2 \mathrm{Nx}$ & 26.3 & 31.2 & 27.6 & 31.4 & 25.0 & 6.7 & -26.3 & -21.6 \\
\hline 3 & $\mathrm{M}$ & 74 & T3N2c & 181.6 & 24.9 & 20.7 & 37.0 & 31.2 & -10.2 & -34.0 & -3.0 \\
\hline 4 & $\mathrm{~F}$ & 66 & T2N2c & 107.2 & 27.8 & 23.5 & 32.0 & 27.9 & 8.6 & -30.1 & -24.9 \\
\hline 5 & $M$ & 57 & T3N0 & 62.5 & 20.7 & 18.0 & 28.1 & 27.8 & $\begin{array}{l}-55.4 \\
\end{array}$ & -57.6 & \begin{tabular}{|l|}
-15.4 \\
\end{tabular} \\
\hline 6 & $\mathrm{M}$ & 67 & T3N2c & 156.2 & 24.5 & 22.7 & 27.6 & 29.4 & -40.1 & -12.7 & -39.8 \\
\hline 7 & $\mathrm{M}$ & 52 & T4N2 & 174.0 & $\mathrm{~N} / \mathrm{A}$ & 21.6 & $\mathrm{~N} / \mathrm{A}$ & 28.7 & -13.2 & N/A & -43.1 \\
\hline 8 & $M$ & 67 & T4N1 & 139.4 & 22.0 & 19.3 & 30.7 & 29.2 & -8.5 & $\begin{array}{l}-49.8 \\
\end{array}$ & \begin{tabular}{|l|}
-57.3 \\
\end{tabular} \\
\hline 9 & $\mathrm{~F}$ & 65 & T3N3 & 237.6 & 24.0 & 20.3 & 42.4 & 31.1 & -56.1 & -24.9 & -30.8 \\
\hline 10 & $F$ & 65 & T4N3 & 257.9 & N/A & 24.6 & $\mathrm{~N} / \mathrm{A}$ & 35.3 & 12.6 & $\mathrm{~N} / \mathrm{A}$ & -18.6 \\
\hline 11 & M & 50 & T4N2c & 434.6 & N/A & 17.8 & N/A & 36.3 & -62.1 & N/A & -54.2 \\
\hline 12 & $\mathrm{M}$ & 53 & T3N0 & 14.4 & 16.6 & 23.3 & 41.4 & 24.3 & -14.1 & $\begin{array}{l}-44.8 \\
\end{array}$ & -5.2 \\
\hline 13 & $\mathrm{M}$ & 73 & T3N2c & 147.0 & 29.5 & 29.3 & 54.6 & 32.2 & -32.7 & $\begin{array}{l}-54.9 \\
\end{array}$ & 5.2 \\
\hline
\end{tabular}


M: male; F: female; CT0: initial planning; $\mathrm{CTV}_{70}$ : clinical target volume receiving $70 \mathrm{~Gy}$; PGs: parotid glands; ILP: ipsilateral PGs; CLP: contralateral PGs; Dmean: mean dose at initial planning; N/A: not applicable (PGs included in the CTV); Volume variation: volume difference between the planning CT and the last CT (in percentage) 
Table 2: Best ART scenario of replanning(s) to spare the PG considering the average PG dose to cumulate the dose in the PGs

\begin{tabular}{|c|c|c|c|c|}
\hline $\begin{array}{l}\text { Number of } \\
\text { replannings }\end{array}$ & $\begin{array}{l}\text { Best week(s) for } \\
\text { replannings }\end{array}$ & $\begin{array}{c}\text { Average CL PG } \\
\text { dose (Gy) } \\
\text { mean (min - max) }\end{array}$ & $\begin{array}{c}\text { Average IL PG } \\
\text { dose (Gy) } \\
\text { mean (min - max) }\end{array}$ & $\begin{array}{l}\text { Average D98\% of } \\
\qquad \mathrm{CTV}_{70}(\mathrm{~Gy}) \\
\text { mean (min - max) }\end{array}$ \\
\hline 0 & $\mathrm{~N} / \mathrm{A}$ & $31.7(18.1-44.3)$ & $37.4(29.4-57.2)$ & $67,8(66,1-69,5)$ \\
\hline 1 & $\mathrm{w}_{1}$ & $29.6(18.2-39.3) *$ & $35.2(24.4-50.6) *$ & $68,1(66,3-69,7)$ \\
\hline 2 & $\mathrm{w}_{1}, \mathrm{w}_{5}$ & $28.6(18.2-35.8) *$ & $34.8(24.0-50.7) *$ & $68,4(67,3-69,4) *$ \\
\hline 3 & $\mathrm{w}_{1}, \mathrm{w}_{2}, \mathrm{w}_{5}$ & $28.3(18.3-38.8) *$ & $34.8(24.2-51.4) *$ & $68,4(67,3-69,5) *$ \\
\hline 4 & $\mathrm{w}_{1}, \mathrm{w}_{2}, \mathrm{w}_{4}, \mathrm{w}_{5}$ & $28.1(18.0-37.1) *$ & $34.7(24.3-51.2) *$ & $68,7(67,5-69,7) *$ \\
\hline 5 & $\mathrm{w}_{1}, \mathrm{w}_{2}, \mathrm{w}_{4}, \mathrm{w}_{5}, \mathrm{w}_{6}$ & $28.0(18.0-37.1) *$ & $34.6(25.3-51.3) *$ & $68,7(67,6-69,7) *$ \\
\hline 6 & $\mathrm{w}_{1}, \mathrm{w}_{2}, \mathrm{w}_{3}, \mathrm{w}_{4}, \mathrm{w}_{5}, \mathrm{w}_{6}$ & $28.0(17.9-36.4) *$ & $34.7(26.1-51.2) *$ & $68,9(67,7-70,0) *$ \\
\hline
\end{tabular}

W: week; N/A: not applicable; PG: parotid gland, CL: contralateral, IL: ipsilateral

The given PG doses correspond to the PG mean dose (Dmean). For each patient and each replanning scenario, the PG dose delivered during the whole treatment was estimated by calculating Dmean average.

The CL and IL PG mean (range) planning Dmean were 30.0 Gy (24.3 - 36.4 Gy) and 35.8 Gy (27.7 - 54.6 Gy) respectively (Table 1). The mean (range) planning $\mathrm{CTV}_{70}$ D98\% was 69.1 Gy (68.2 - 70.0 Gy). Compared to the non-ART scenario ( 0 replanning), increasing the number of replannings significantly decreased the PG mean dose $\left(^{*}\right.$ in the $2^{\text {nd }}$ and $3^{\text {rd }}$ column) and increased the CTV coverage $\left(*\right.$ in the $5^{\text {th }}$ column) $(*$ Wilcoxon test with $\mathrm{p}<0.05)$. 
Table 3 (Supplementary material): Best ART scenario of replanning(s) to spare the PG, considering deformable image registration to cumulate the dose in the PGs

\begin{tabular}{|c|c|c|c|}
\hline $\begin{array}{l}\text { Number of } \\
\text { replannings }\end{array}$ & $\begin{array}{l}\text { Best week(s) for } \\
\text { replannings }\end{array}$ & $\begin{array}{l}\text { DIR Cumulated CL PG dose } \\
\qquad(G y) \\
\text { mean }(\min -\max )\end{array}$ & $\begin{array}{l}\text { DIR Cumulated IL PG dose } \\
\qquad(\text { Gy) } \\
\text { mean (min - max) }\end{array}$ \\
\hline 0 & $\mathrm{~N} / \mathrm{A}$ & $31.6(19.3-41.9)$ & $36.6(27.3-57.6)$ \\
\hline 1 & $\mathrm{w}_{1}$ & $29.5(19.4-37.7)$ & $34.3(24.9-50.6) *$ \\
\hline 2 & $\mathrm{w}_{1}, \mathrm{w}_{5}$ & $28.3(19.5-33.6) *$ & $34.0(24.6-50.8) *$ \\
\hline 3 & $\mathrm{w}_{1}, \mathrm{w}_{2}, \mathrm{w}_{5}$ & $28.0(19.5-35.8) *$ & $34.0(25.3-51.5) *$ \\
\hline 4 & $\mathrm{w}_{1}, \mathrm{w}_{2}, \mathrm{w}_{4}, \mathrm{w}_{5}$ & $27.8(19.2-33.7) *$ & $34.0(25.4-51.4) *$ \\
\hline 5 & $\mathrm{w}_{1}, \mathrm{w}_{2}, \mathrm{w}_{4}, \mathrm{w}_{5}, \mathrm{w}_{6}$ & $27.7(19.2-33.7) *$ & $33.8(25.9-51.4) *$ \\
\hline 6 & $\mathrm{w}_{1}, \mathrm{w}_{2}, \mathrm{w}_{3}, \mathrm{w}_{4}, \mathrm{w}_{5}, \mathrm{w}_{6}$ & $27.7(19.2-32.7) *$ & $34.0(25.9-51.3) *$ \\
\hline
\end{tabular}

W: week; N/A: not applicable; PG: parotid gland, CL: contralateral, IL: ipsilateral

The given PG doses correspond to the PG mean dose (Dmean). For each patient and each replanning scenario, the PG dose delivered during the whole treatment was estimated by deformable image registration.

Compared to the non-ART scenario (0 replanning), increasing the number of replannings significantly decreased the PG mean dose $\left(*\right.$ in the $2^{\text {nd }}$ and $3^{\text {rd }}$ column). 
Figure 1: Study workflow

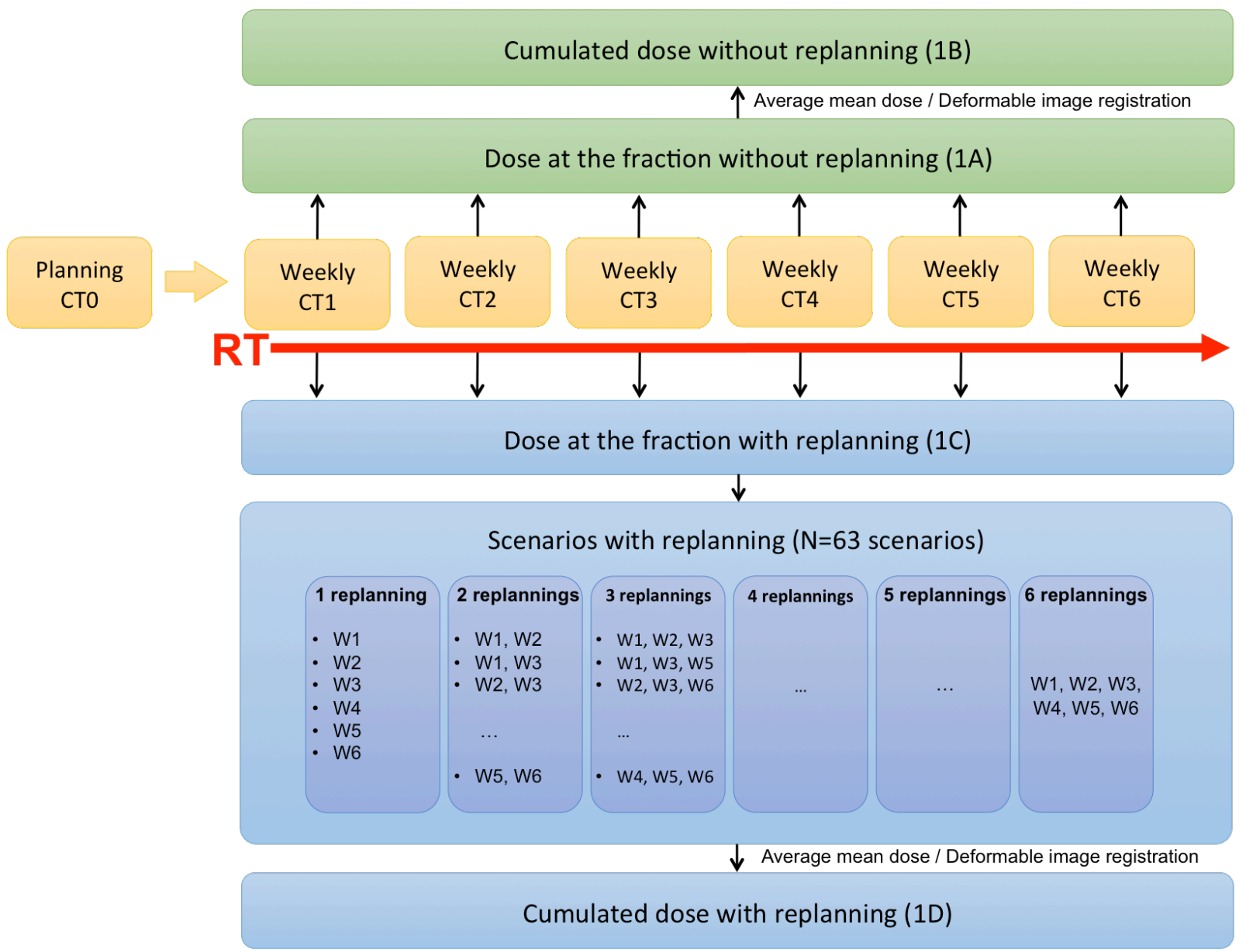

CT: computed tomography; W: week

The weekly doses were recalculated without replanning (step 1A) or with replanning (step 1C). The cumulated doses without replanning (step 1B) and for each scenario of replanning (step 1D) were calculated by average mean dose and deformable image registration, and then the doses were compared 
Figure 2: Benefit of replanning(s) to spare the PGs for each ART scenario, considering the average

\section{PG mean doses}

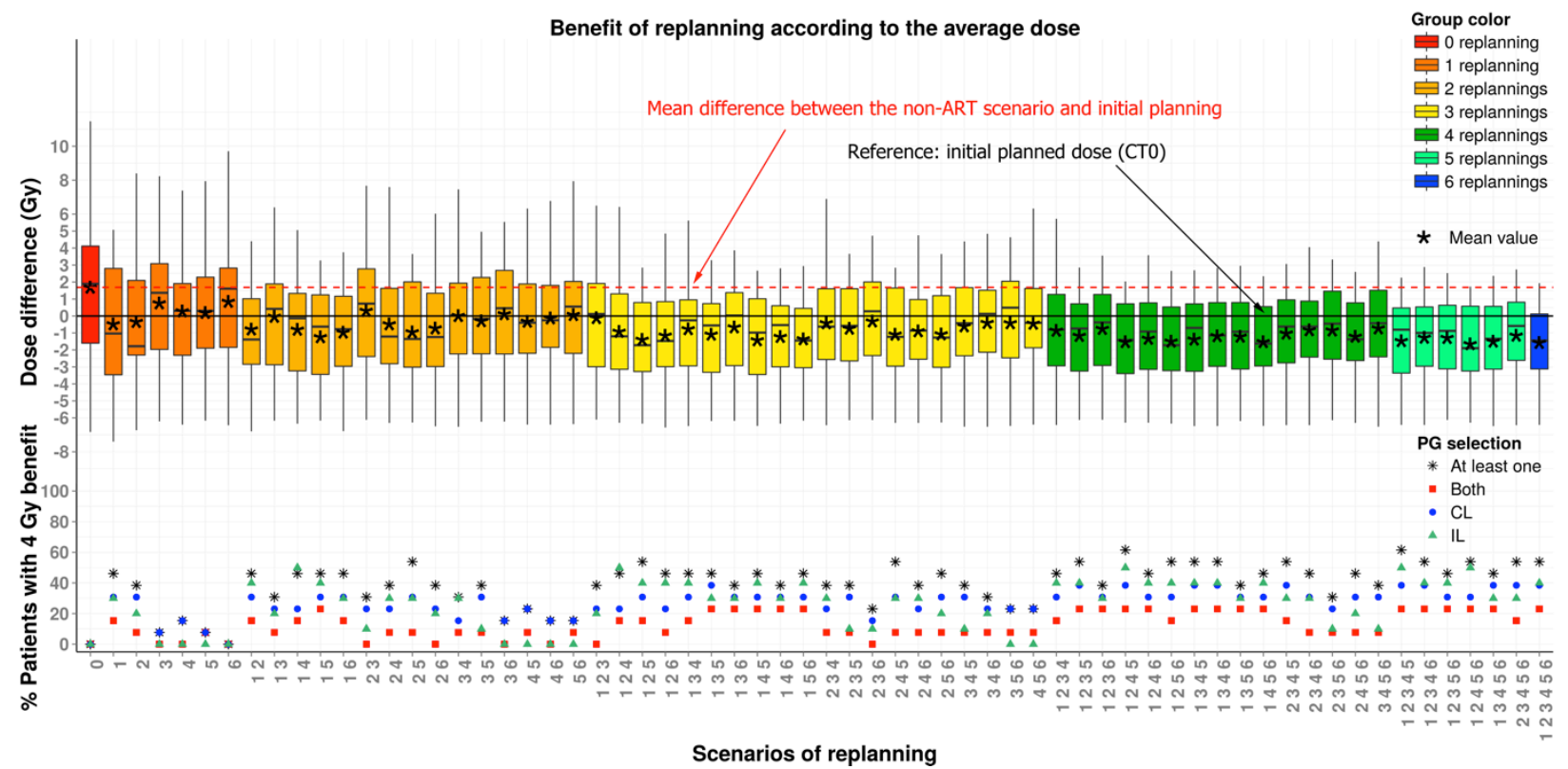

ART: adaptive radiation therapy; PG: parotid gland; CL: contralateral; IL: ipsilateral

Each boxplot represents the difference between the cumulated dose and the planned dose for each of the 63 ART scenarios and the 23 parotid glands (PG). Each scenario is identified by the week(s) of replanning(s), ordered by increasing number of replannings (CT1 to CT6).

The limits of each box represent the $25^{\text {th }}$ and $75^{\text {th }}$ percentiles, the whisker represents the most extreme data points and the black line represents the median. The horizontal black line represents the reference of the dose differences, which is the planned dose on CT0. The red dotted line represents the median cumulated dose difference of the cohort (from the non-ART or " 0 replanning" boxplot).

The color points in the inferior part represent the percentage of patients who had, for each scenario, a benefit superior to 4 Gy compared to the delivered dose (non-ART) for both PGs in black, at least one PG in green, only the IL PGs in blue and only the CL PGs in red compared to the cumulated dose. 
Figure 3: Percentage of patients with a PG benefit of more than a given threshold dose for the threereplannings scenario (weeks 1-2-5), considering the average PG mean doses to cumulate the dose in the PGs

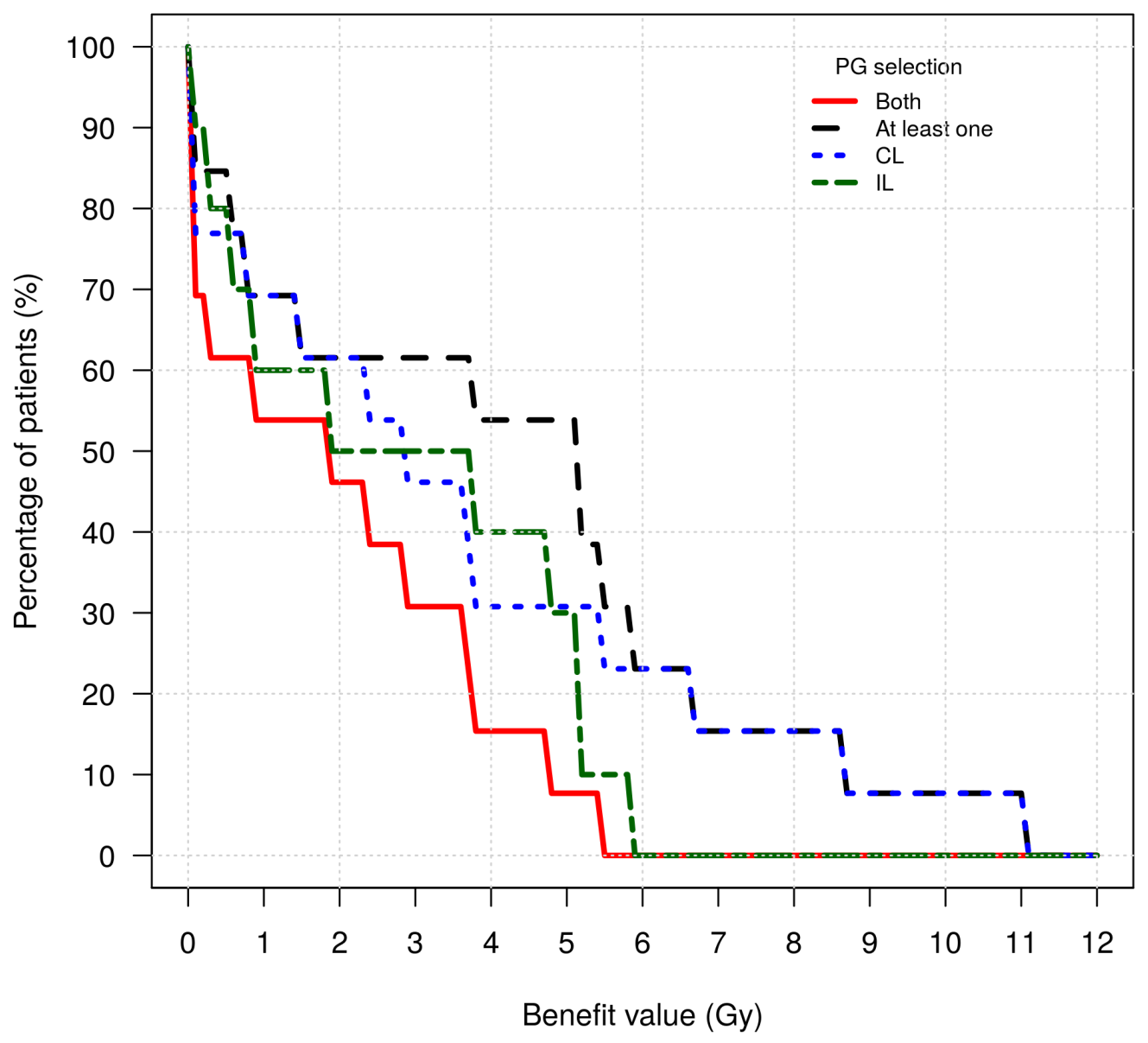

Each curve represents the percentage of patients with a PG benefit (mean dose) of more than a given threshold dose for both parotid glands (in red), at least one (in black), the contralateral one (in blue) and the ipsilateral one (in green). 
Figure 4 (Supplementary material): Benefit of replanning(s) to spare the PGs for each ART scenario, considering DIR-based cumulated PG doses

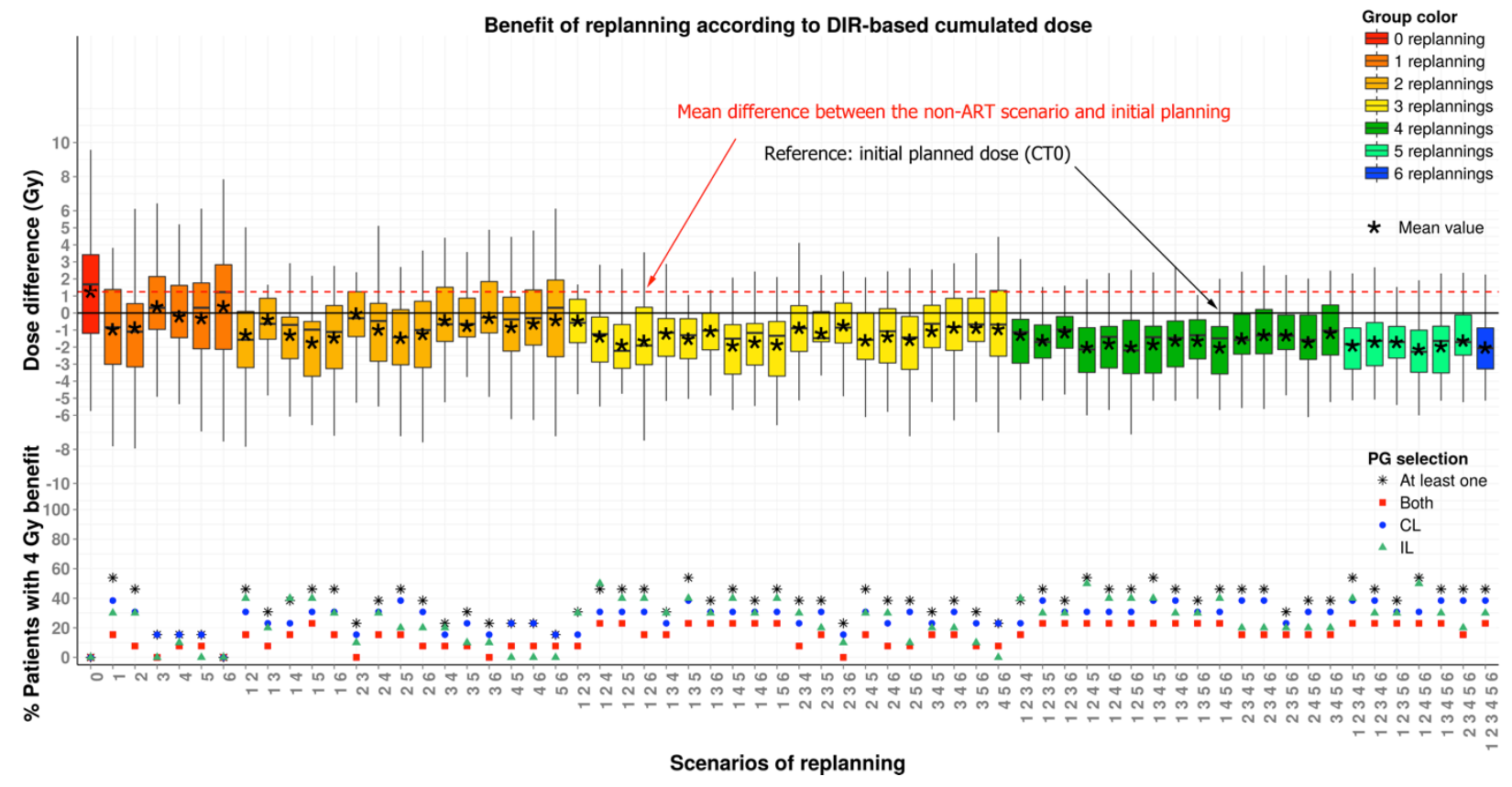

ART: adaptive radiation therapy; PG: parotid gland; CL: contralateral; IL: ipsilateral

Each boxplot represents the difference between the cumulated dose and the planned dose for each of the 63 ART scenarios and the 23 parotid glands (PG). Each scenario is identified by the week(s) of replanning(s), ordered by increasing number of replannings (CT1 to CT6).

The limits of each box represent the $25^{\text {th }}$ and $75^{\text {th }}$ percentiles, the whisker represents the most extreme data points and the black line represents the median. The horizontal black line represents the reference of the dose differences, which is the planned dose on CT0. The red dotted line represents the median cumulated dose difference of the cohort (from the non-ART or "0 replanning" boxplot).

The color points in the inferior part represent the percentage of patients who had, for each scenario, a benefit superior to 4 Gy compared to the delivered dose (non-ART) for both PGs in black, at least one PG in green, only the IL PGs in blue and only the CL PGs in red compared to the cumulated dose. 
Figure 5 (Supplementary material): Percentage of patients with a PG benefit of more than a given threshold dose for the three-replannings scenario (weeks 1-2-5), considering DIR-based cumulated PG doses

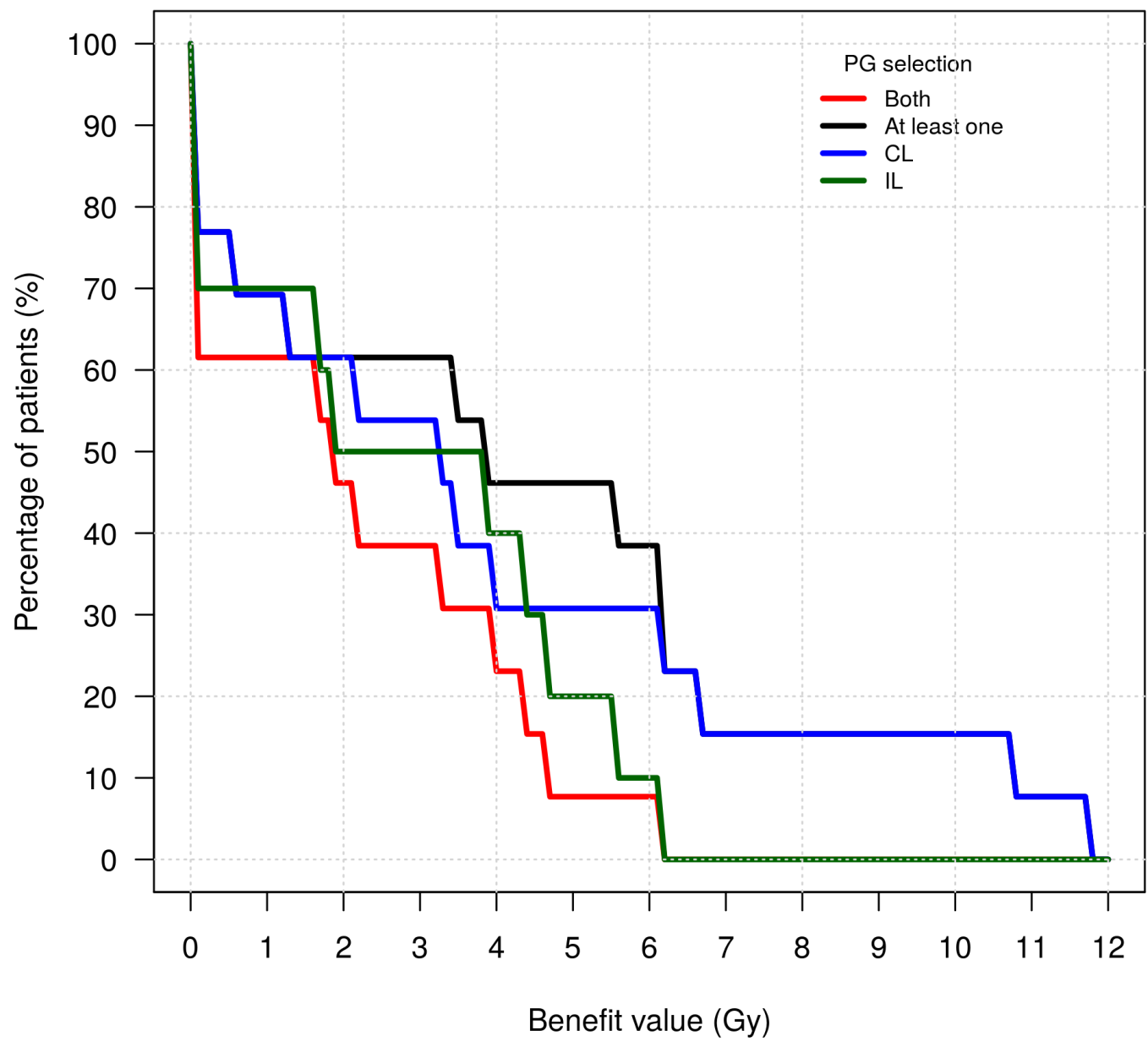

Each curve represents the percentage of patients with a PG benefit (mean dose) of more than a given threshold dose for both parotid glands (in red), at least one (in black), the contralateral one (in blue) and the ipsilateral one (in green). 


\section{References}

1. Kam MK, Leung S-F, Zee B, Chau RM, Suen JJ, Mo F, Lai M, Ho R, Cheung K-y, Brian K. Prospective randomized study of intensity-modulated radiotherapy on salivary gland function in early-stage nasopharyngeal carcinoma patients. Journal of Clinical Oncology 2007;25:4873-4879.

2. Nutting CM, Morden JP, Harrington KJ, Urbano TG, Bhide SA, Clark C, Miles EA, Miah AB, Newbold K, Tanay $M$. Parotid-sparing intensity modulated versus conventional radiotherapy in head and neck cancer (parsport): A phase 3 multicentre randomised controlled trial. The lancet oncology 2011;12:127-136.

3. Pow EH, Kwong DL, McMillan AS, Wong MC, Sham JS, Leung LH, Leung WK. Xerostomia and quality of life after intensity-modulated radiotherapy vs. Conventional radiotherapy for early-stage nasopharyngeal carcinoma: Initial report on a randomized controlled clinical trial. International journal of radiation oncology, biology, physics 2006;66:981-991.

4. Barker JL, Garden AS, Ang KK, O'Daniel JC, Wang H, Court LE, Morrison WH, Rosenthal DI, Chao KC, Tucker SL. Quantification of volumetric and geometric changes occurring during fractionated radiotherapy for head-and-neck cancer using an integrated ct/linear accelerator system. International Journal of Radiation Oncology* Biology* Physics 2004;59:960-970.

5. Duma M, Kampfer S, Schuster T, Winkler C, Geinitz H. Adaptive radiotherapy for soft tissue changes during helical tomotherapy for head and neck cancer. Strahlentherapie und Onkologie 2012;188:243-247.

6. Nishi T, Nishimura $Y$, Shibata T, Tamura M, Nishigaito N, Okumura M. Volume and dosimetric changes and initial clinical experience of a two-step adaptive intensity modulated radiation therapy (imrt) scheme for head and neck cancer. Radiotherapy and Oncology 2013;106:85-89.

7. Lai Y-L, Yang S-N, Liang J-A, Wang Y-C, Yu C-Y, Su C-H, Chen S-W. Impact of body-mass factors on setup displacement in patients with head and neck cancer treated with radiotherapy using daily on-line image guidance. Radiation oncology 2014;9:19.

8. Castelli J, Simon A, Louvel G, Henry O, Chajon E, Nassef M, Haigron P, Cazoulat G, Ospina J, Jegoux F, Benezery K, de Crevoisier R. Impact of head and neck cancer adaptive radiotherapy to spare the parotid glands and decrease the risk of xerostomia. Radiat Oncol 2015;10:6.

9. You SH, Kim SY, Lee CG, Keum KC, Kim JH, Lee IJ, Kim YB, Koom WS, Cho J, Kim SK. Is there a clinical benefit to adaptive planning during tomotherapy in patients with head and neck cancer at risk for xerostomia? American journal of clinical oncology 2012;35:261-266.

10. Hansen EK, Bucci MK, Quivey JM, Weinberg V, Xia P. Repeat ct imaging and replanning during the course of imrt for head-and-neck cancer. International Journal of Radiation Oncology* Biology* Physics 2006;64:355-362.

11. Lee C, Langen KM, Lu W, Haimerl J, Schnarr E, Ruchala KJ, Olivera GH, Meeks SL, Kupelian PA, Shellenberger TD. Assessment of parotid gland dose changes during head and neck cancer radiotherapy using daily megavoltage computed tomography and deformable image registration. International Journal of Radiation Oncology* Biology* Physics 2008;71:1563-1571.

12. Chambers MS, Rosenthal DI, Weber RS. Radiation - induced xerostomia. Head \& neck 2007;29:58-63.

13. Wu Q, Chi Y, Chen PY, Krauss DJ, Yan D, Martinez A. Adaptive replanning strategies accounting for shrinkage in head and neck imrt. International journal of radiation oncology, biology, physics 2009;75:924-932. 
14. Olteanu LA, Madani I, De Neve W, Vercauteren T, De Gersem W. Evaluation of deformable image coregistration in adaptive dose painting by numbers for head-and-neck cancer. International journal of radiation oncology, biology, physics 2012;83:696-703.

15. Olteanu LA, Berwouts D, Madani I, De Gersem W, Vercauteren T, Duprez F, De Neve W. Comparative dosimetry of three-phase adaptive and non-adaptive dose-painting imrt for head-and-neck cancer. Radiotherapy and oncology : journal of the European Society for Therapeutic Radiology and Oncology 2014.

16. Fung WWK, Wu VWC, Teo PML. Developing an adaptive radiation therapy strategy for nasopharyngeal carcinoma. Journal of radiation research 2014;55:293-304.

17. Berwouts D, Olteanu LA, Duprez F, Vercauteren T, De Gersem W, De Neve W, Van de Wiele C, Madani I. Three-phase adaptive dose-painting-by-numbers for head-and-neck cancer: Initial results of the phase i clinical trial. Radiotherapy and Oncology 2013;107:310-316.

18. Budach W, Bolke E, Fietkau R, Buchali A, Wendt TG, Popp W, Matuschek C, Sack H. Evaluation of time, attendance of medical staff, and resources during radiotherapy for head and neck cancer patients: The degro-quiro trial. Strahlentherapie und Onkologie : Organ der Deutschen Rontgengesellschaft [et al] 2011;187:449-460.

19. Daisne J-F, Blumhofer A. Atlas-based automatic segmentation of head and neck organs at risk and nodal target volumes: A clinical validation. Radiation oncology 2013;8:154.

20. Lee N, Chuang C, Quivey JM, Phillips TL, Akazawa P, Verhey LJ, Xia P. Skin toxicity due to intensitymodulated radiotherapy for head-and-neck carcinoma. International Journal of Radiation Oncology* Biology* Physics 2002;53:630-637.

21. Eisbruch A, Harris J, Garden AS, Chao CK, Straube W, Harari PM, Sanguineti G, Jones CU, Bosch WR, Ang KK. Multi-institutional trial of accelerated hypofractionated intensity-modulated radiation therapy for early-stage oropharyngeal cancer (rtog 00-22). International Journal of Radiation Oncology* Biology* Physics 2010;76:1333-1338.

22. Cazoulat G, Simon A, Dumenil A, Gnep K, De Crevoisier R, Acosta O, Haigron P. Surface-constrained nonrigid registration for dose monitoring in prostate cancer radiotherapy. Medical Imaging, IEEE Transactions on 2014;33:1464-1474.

23. Rigaud B, Simon A, Castelli J, Gobeli M, Ospina Arango JD, Cazoulat G, Henry O, Haigron P, De Crevoisier R. Evaluation of deformable image registration methods for dose monitoring in head and neck radiotherapy. BioMed research international 2015;2015:726268.

24. Dice LR. Measures of the amount of ecologic association between species. Ecology 1945;26:297-302.

25. Castadot $P$, Geets $X$, Lee JA, Grégoire V. Adaptive functional image-guided imrt in pharyngo-laryngeal squamous cell carcinoma: Is the gain in dose distribution worth the effort? Radiotherapy and Oncology 2011;101:343-350.

26. Schwartz DL, Garden AS, Shah SJ, Chronowski G, Sejpal S, Rosenthal DI, Chen Y, Zhang Y, Zhang L, Wong P-F. Adaptive radiotherapy for head and neck cancer-dosimetric results from a prospective clinical trial. Radiotherapy and Oncology 2013;106:80-84.

27. Fung WWK, Wu VWC, Teo PML. Dosimetric evaluation of a three-phase adaptive radiotherapy for nasopharyngeal carcinoma using helical tomotherapy. Medical Dosimetry 2012;37:92-97.

28. Ahn PH, Chen C-C, Ahn Al, Hong L, Scripes PG, Shen J, Lee C-C, Miller E, Kalnicki S, Garg MK. Adaptive planning in intensity-modulated radiation therapy for head and neck cancers: Single-institution experience and clinical implications. International Journal of Radiation Oncology* Biology* Physics 2011;80:677-685.

29. Capelle L, Mackenzie M, Field C, Parliament M, Ghosh S, Scrimger R. Adaptive radiotherapy using helical tomotherapy for head and neck cancer in definitive and postoperative settings: Initial results. Clinical Oncology 2012;24:208-215. 
30. Chen AM, Daly ME, Cui J, Mathai M, Benedict S, Purdy JA. Clinical outcomes among patients with head and neck cancer treated by intensity-modulated radiotherapy with and without adaptive replanning. Head \& neck 2013.

31. Zhao L, Wan Q, Zhou Y, Deng X, Xie C, Wu S. The role of replanning in fractionated intensity modulated radiotherapy for nasopharyngeal carcinoma. Radiotherapy and Oncology 2011;98:2327.

32. Wang R, Zhang S, Zhou L, Zhang G, Yu H, Lin X, Lin S. Volume and dosimetric variations during twophase adaptive intensity-modulated radiotherapy for locally advanced nasopharyngeal carcinoma. Bio-medical materials and engineering 2013;24:1217-1225.

33. Yang $\mathrm{H}, \mathrm{Hu} \mathrm{W}$, Wang $\mathrm{W}$, Chen $\mathrm{P}$, Ding $\mathrm{W}$, Luo $\mathrm{W}$. Replanning during intensity modulated radiation therapy improved quality of life in patients with nasopharyngeal carcinoma. International journal of radiation oncology, biology, physics 2013;85:e47-54.

34. Huang H, Lu H, Feng G, Jiang H, Chen J, Cheng J, Pang Q, Lu Z, Gu J, Peng L. Determining appropriate timing of adaptive radiation therapy for nasopharyngeal carcinoma during intensity-modulated radiation therapy. Radiation Oncology 2015;10:192.

35. Ho KF, Marchant T, Moore C, Webster G, Rowbottom C, Penington H, Lee L, Yap B, Sykes A, Slevin N. Monitoring dosimetric impact of weight loss with kilovoltage (kv) cone beam ct (cbct) during parotid-sparing imrt and concurrent chemotherapy. International Journal of Radiation Oncology* Biology* Physics 2012;82:e375-e382.

36. Wang W, Yang H, Hu W, Shan G, Ding W, Yu C, Wang B, Wang X, Xu Q. Clinical study of the necessity of replanning before the 25th fraction during the course of intensity-modulated radiotherapy for patients with nasopharyngeal carcinoma. International Journal of Radiation Oncology* Biology* Physics 2010;77:617-621.

37. Bhandari V, Patel P, Gurjar OP, Gupta KL. Impact of repeat computerized tomography replans in the radiation therapy of head and neck cancers. Journal of Medical Physics 2014;39:164.

38. Bhide SA, Davies M, Burke K, McNair HA, Hansen V, Barbachano Y, El-Hariry I, Newbold K, Harrington $\mathrm{KJ}$, Nutting CM. Weekly volume and dosimetric changes during chemoradiotherapy with intensity-modulated radiation therapy for head and neck cancer: A prospective observational study. International Journal of Radiation Oncology* Biology* Physics 2010;76:1360-1368.

39. Hunter KU, Fernandes LL, Vineberg KA, McShan D, Antonuk AE, Cornwall C, Feng M, Schipper MJ, Balter JM, Eisbruch A. Parotid glands dose-effect relationships based on their actually delivered doses: Implications for adaptive replanning in radiation therapy of head-and-neck cancer. International journal of radiation oncology, biology, physics 2013;87:676-682.

40. Sanguineti G, Ricchetti F, Wu B, McNutt T, Fiorino C. Parotid gland shrinkage during imrt predicts the time to xerostomia resolution. Radiation Oncology 2015;10:1.

41. van Luijk P, Pringle S, Deasy JO, Moiseenko VV, Faber H, Hovan A, Baanstra M, van der Laan HP, Kierkels RG, van der Schaaf A. Sparing the region of the salivary gland containing stem cells preserves saliva production after radiotherapy for head and neck cancer. Science translational medicine 2015;7:305ra147-305ra147.

42. Brouwer CL, Steenbakkers RJ, Langendijk JA, Sijtsema NM. Identifying patients who may benefit from adaptive radiotherapy: Does the literature on anatomic and dosimetric changes in head and neck organs at risk during radiotherapy provide information to help? Radiotherapy and Oncology 2015;115:285-294. 